\title{
Vestibular Screening in Pediatric Patients with Otitis Media
}

DOI: $10.3766 /$ jaaa. 18101

\author{
Sonia Kim Rehagen* \\ Maureen Valente* \\ Judith E. C. Lieu*
}

\begin{abstract}
Background: Otitis media with effusion (OME) is a common cause of vestibular disturbances in children. However, young children often lack the language to express their symptoms, and it is uncommon to screen children for vestibular impairments.

Purpose: The purpose of this study was to develop a screening protocol for children presenting with OME to determine if diagnostic vestibular testing is necessary.
\end{abstract}

Research Design: Children with normal hearing $(\mathrm{NH})$, sensorineural hearing loss (SNHL), and conductive hearing loss $(\mathrm{CHL})$ due to OME participated in a vestibular screening.

Study Sample: There were 30 participants, ages four to eight years, ranging from 48 to 101 months included in the study: 10 with $\mathrm{NH}, 11$ with $\mathrm{CHL}$ due to OME, and 9 with SNHL.

Data Collection and Analysis: The vestibular screening consists of patient and parent questionnaires and a functional evaluation. The tests examined coordination, balance, oculomotor function, and nystagmus.

Results: Those with CHL were significantly more likely to display abnormal smoothness of pursuit, as measured with observations for rapid tracking, absence of or delayed saccades, and overshoot, than those with $\mathrm{NH}$ or SNHL $(p=0.012)$. Parents of children with $\mathrm{CHL}$ due to OME were more likely to report their child experiencing middle ear pressure than the parents of children with NH or SNHL $(p=0.010)$. In addition, children with $\mathrm{CHL}$ were less likely to report hearing loss than those with $\mathrm{NH}$ or SNHL. Parent and patient report were not found to be reliable indicators of vestibular disturbances.

Conclusions: This pilot study suggests that children with $\mathrm{CHL}$ due to OME present with more oculomotor abnormalities than their peers with $\mathrm{NH}$. Further research is necessary to determine validity and reliability of the findings for this present study.

Key Words: balance, otitis media with effusion, vestibular screening

Abbreviations: BOT-2 = Bruininks-Oseretsky Test of Motor Proficiency-2; $\mathrm{CHL}=$ conductive hearing loss; $\mathrm{NH}=$ normal hearing; $\mathrm{OME}=$ otitis media with effusion; $\mathrm{SD}=$ standard deviation; $\mathrm{SNHL}=$ sensorineural hearing loss; VOR = vestibulo-ocular reflex

\section{INTRODUCTION}

\section{Otitis Media}

Otitis media with effusion (OME) is an inflammation of the middle ear in which fluid is present. It is a common chronic disease of childhood, second only to upper respiratory tract infections (Koyuncu et al, 1999). Significant costs are associated with the care and management of OME. According to the United States Department of Health and Human Services, the cost of treating $\mathrm{OME}$ in children in 2006 was

*Washington University School of Medicine Program in Audiology and Communication Sciences, St. Louis, M0 and Department of Otolaryngology-Head and Neck Surgery, Washington University School of Medicine, St. Louis, MO

Corresponding author: Sonia Kim Rehagen, Lucile Packard Children's Hospital at Stanford Palo Alto, CA 94085; Email: sonia.kim@wustl.edu

Presented at Program in Audiology and Communication Sciences Student Research Colloquium (May 2017) and Washington University School of Medicine Otolaryngology Research Seminar (December 2017). 
$\$ 2.8$ billion, excluding over-the-counter medications (Soni, 2006).

OME can impact health, general development, and cognitive outcomes in children. Most notably, OME is accompanied by hearing loss in up to $60 \%$ of children as a result of compromised conduction due to middle ear fluid (Gravel and Wallace, 2000). A longitudinal study by Bennett et al (2001) found that children with chronic OME exhibited, on average, a two-year delay in mean reading scores than those with normal hearing $(\mathrm{NH})$. The decreased access to sound presents a significant barrier for children's academic and communication abilities.

Patients with OME can experience dizziness and/or vertigo, indicating that inflammation of the middle ear also affects the vestibular system given the anatomical proximity of the otolithic organ to the cochlea (da Costa Monsanto et al, 2016). In fact, otitis media has been considered the most common cause of vestibular disturbances in children (Koyuncu et al, 1999). However, younger children often cannot articulate their symptoms, complicating identification of the source and severity of the problem (McCaslin et al, 2015).

\section{Vestibular System}

The vestibular system is involved in postural control and gaze stabilization (Rine and Wiener-Vacher, 2013). The otolithic organs detect linear accelerations, which enable control of head position on the body as well as vestibulo-ocular reflex (VOR). The VOR involves the ability to maintain a stable image on the fovea during head movement. Visual acuity is similar during head movement as when it is stationary, but there is a degradation of dynamic visual acuity with vestibular disturbances (Rine and Wiener-Vacher, 2013). In addition, the vestibular system processes information for coordination and execution of basic motor reflexes to complex coordination of the eyes, head, limbs, and trunk (Day and Fitzpatrick, 2005). As a result, disruption to any part of this multisensory system can lead to impairments of balance and gaze stability (Rine and Weiner-Vacher, 2013).

\section{Pediatric Vestibular Disturbances}

Vestibular disturbances delay the development of motor skills that require dynamic balance because of poor static balance and incoordination (McCaslin et al, 2015). Several studies have reported delayed development of walking and balance in children with vestibular dysfunction (Tsuzuku and Kaga, 1992; Deitz et al, 1996; Wiener-Vacher et al, 2012). Schaaf (1985) reported significantly higher incidence of vestibular disorders in children with history of OME than those without OME. Childhood vestibular disturbances can lead to detrimental effects on motor development.

Pediatric vestibular testing is rarely conducted despite audiologic advances in adapting assessment techniques for children and the growing evidence of childhood vestibular dysfunction. Barriers to testing include poor patient report, cost, and time associated with testing. Consequently, pediatric vestibular dysfunction is underdiagnosed and treatment is not always provided to address development impairments (Braswell and Rine, 2006).

\section{Present Literature and Clinical Practice}

The literature regarding pediatric vestibular disturbances and clinically used assessment tools is limited. However, recent efforts to assess vestibular function in the pediatric population have yielded significant progress (Sheykholeslami et al, 2005; Valente, 2007). Several studies support the use of cervical vestibularevoked myogenic potential in children to diagnose vestibular impairment (Picciotti et al, 2007; Zhou et al, 2014).

There are assessments to evaluate vestibular function without electrophysiological measures. One commonly used assessment is the Bruininks-Oseretsky Test of Motor Proficiency, Second Edition (BOT-2). The BOT-2 is a standardized, norm-referenced measure used to assess fine and gross motor skills in children ages four through 21 years (Deitz et al, 2007). The balance subtest evaluates motor skills required to maintain posture while standing and walking (Wolter et al, 2016). The high accessibility, standardization, and time-efficient nature make the BOT-2 a clinically useful tool to assess balance. Of note, the BOT-2 addresses the vestibulospinal reflex, but it does not evaluate VOR, a necessary component for a comprehensive vestibular assessment measure.

The purpose of this pilot study was to compare children with conductive hearing loss (CHL) due to OME with children with $\mathrm{NH}$ and sensorineural hearing loss (SNHL) to determine if a screening can identify vestibular disturbances. An efficient screen routinely administered for children with OME at the otolaryngologist's office can help refer those in need of further testing.

\section{MATERIALS AND METHODS}

$T$ he study protocol received institutional review board approval by the Human Research Protection Office at the Washington University School of Medicine in St. Louis. Before testing, written consent and verbal assent were obtained from the guardians and children, respectively. 


\section{Participants}

Participants were recruited through St. Louis Children's Hospital, Children's Specialty Care Center, Central Institute for the Deaf, and Missouri Hands \& Voices.

There were 30 participants, ages four to eight years $($ mean $=5.48$ years, median $=5$ years, mode $=5$ years $)$, included in this study. They were categorized into three groups: NH, CHL due to OME, and SNHL. For the purpose of the pilot study, 30 participants were recruited, including 10 participants within each group. The inclusion criteria were as follows.

The NH group demonstrated pure-tone thresholds less than or equal to $20 \mathrm{~dB}$ HL from 500 through $8000 \mathrm{~Hz}$. Children with CHL due to OME had active unilateral or bilateral OME confirmed through their medical charts. An audiogram was obtained within three months of the screen. CHL is defined as an airbone gap exceeding $10 \mathrm{~dB}$ HL at 2 or more frequencies from 500 through $4000 \mathrm{~Hz}$ and type B tympanogram. Those in the SNHL group had a permanent hearing loss of slight to profound degree, verified through an audiogram dated within a year at the time of testing. Normal middle ear status was affirmed through the audiogram but could not be reassessed at the screening because of limited equipment.

Exclusion criteria included physical disabilities, cognitive delays, or learning disabilities as reported by the parent, and non-native English speakers. Additional exclusion criteria for the CHL due to OME group included CHL due to anatomical malformations.

\section{Equipment}

The equipment was kept to a minimum for accessibility during office visits. Equipment included an Airex balance pad $(50 \mathrm{~cm} \times 41 \mathrm{~cm} \times 6 \mathrm{~cm})$, measuring tape, and painter's tape for the functional test; $14^{\prime \prime} \times 9^{\prime \prime}$ Snellen Eye Chart to test visual acuity; a ball to test for nystagmus and smooth pursuit; two different color toys for visual tracking during saccades; At-Metronome app to maintain consistent timing during postheadshake nystagmus and dynamic visual acuity; parent and patient questionnaire. The setup for the BOT-2 involved placement of painter's tape extending 10 feet from the wall. The top of the Snellen Eye Chart was mounted on a wall five feet above the floor.

\section{Procedure}

All tests were performed in a private room in the otolaryngology clinic. The parent completed a brief questionnaire consisting of questions about the child's birth complications, symptoms associated with dizziness, and motor milestones. The examiner verbally ad- ministered the patient questionnaire to the participant; the questionnaire was a modified version of the Pediatric Vestibular Symptom Questionnaire. The Pediatric Vestibular Symptom Questionnaire includes questions about vestibular symptom frequency within the past month and is created for children aged 6 to 17 years. The current patient questionnaire used simplified vocabulary to accommodate younger participants.

The balance test consisted of a modified balance subtest of the BOT-2, summarized in Table 1. Standing on one leg on a balance beam with eyes open and eyes closed and standing heel-to-toe on a balance beam were substituted with standing on foam on one leg-eyes open, to reduce equipment. Raw scores are obtained for each item for the number of steps or number of seconds an activity is sustained. The item raw score is then converted to a point score using the BOT-2 Record Form (Deitz et al, 2007).

The nystagmus/oculomotor tests of the Ten Minute Examination of the Dizzy Patient were included for this study (Goebel, 2001). This examination was originally designed for adults but was adapted by the authors to administer to children. The nystagmus/oculomotor test is summarized in Table 2. The tests included observations for spontaneous nystagmus, gaze nystagmus, and post-headshake nystagmus; smooth pursuit, with observations for smoothness of pursuit during eye movement; saccades, with observations for rapid tracking, delayed or absent saccades, and overshoot; and dynamic visual acuity, which examines the VOR. Observations were made by the tester for nystagmus and oculomotor abnormalities. To reduce the risk of fatigue on any portion of the test, half of the participants within each group were randomly selected to do the balance test first and the other half the nystagmus/oculomotor test first.

\section{Statistics}

Statistical analysis was performed using SPSS Statistics version 23 (IBM, Armonk, NY). A one-way

Table 1. Summary of Balance Test (Modified BruininksOseretsky Test of Motor Proficiency Balance Subtest) Used in the Study with the Time or Steps Required for Maximum Points

\begin{tabular}{llc}
\hline Balance Subtest Items & $\begin{array}{c}\text { Eyes Open or } \\
\text { Closed }\end{array}$ & $\begin{array}{c}\text { Maximum } \\
\text { Score }\end{array}$ \\
\hline Standing with feet apart on a & Open & $10 \mathrm{~s}$ \\
line & Closed & $10 \mathrm{~s}$ \\
Walking forward on a line & Open & $6 \mathrm{steps}$ \\
Standing on 1 leg on a line & Open & $10 \mathrm{~s}$ \\
& Closed & $10 \mathrm{~s}$ \\
Walking forward heel-to-toe & Open & 6 steps \\
on a line & & $10 \mathrm{~s}$ \\
Standing on foam* & Open & \\
\hline
\end{tabular}

${ }^{*}$ Modified for balance beam. 
Table 2. Summary of Nystagmus/Oculomotor Test (Modified 10 Minute Examination of the Dizzy Patient) and the Nystagmus Patterns Observed to Determine if "Normal" or "Abnormal" are Described

\begin{tabular}{lll}
\hline Task & \multicolumn{1}{c}{ Normal } & Abnormal \\
\hline Spontaneous nystagmus & No nystagmus & Nystagmus \\
Gaze nystagmus & No nystagmus & Nystagmus \\
Smooth pursuit & Smoothness of Pursuit & Saccadic eye movement \\
Saccades & Small saccade in direction & Delayed saccades, overshoot, and \\
& of movement & disconjugate eye movements \\
Post-headshake nystagmus & No nystagmus & Nystagmus \\
Dynamic visual acuity & $0-3$ lines lost & $>3$ lines lost \\
\hline
\end{tabular}

between-group analysis of variance was conducted to explore the impact of hearing status on the patient questionnaire and the balance test. Tukey's honestly significance difference test was conducted as a post hoc measure. An independent sample $t$-test was conducted to explore the effects of gender and age on the balance test. Pearson's chi-squared test was used to evaluate the impact of hearing status on the nystagmus/oculomotor test and the parent questionnaire. The Fisher's exact test was conducted to explore effect of hearing status on the patient ques- tionnaire. The statistical significance level was set at $p \leq 0.05$.

\section{RESULTS}

\section{Participants}

Thirty participants were included in this study between August 2016 and February 2017. Demographic data are summarized in Table 3 . There were 16 males and 14 females, and the average age was 5.99 years

Table 3. Demographic Information of Participants Included in the Study

\begin{tabular}{|c|c|c|c|c|c|}
\hline$\overline{I D}$ & Age (Years) & Gender & Hearing Status & Average Balance Score & Average Nystagmus/Oculomotor Score \\
\hline$\overline{11}$ & 4.61 & Male & Sensorineural & 3 & 2 \\
\hline 12 & 8.42 & Male & Conductive & 4 & 2 \\
\hline 13 & 7.70 & Female & Sensorineural & 4 & 2 \\
\hline 14 & 5.83 & Female & Conductive & 4 & 2 \\
\hline 15 & 6.02 & Female & Normal & 4 & 2 \\
\hline 16 & 5.34 & Male & Conductive & 3 & 2 \\
\hline 17 & 7.92 & Female & Normal & 4 & 2 \\
\hline 18 & 6.96 & Female & Normal & 4 & 2 \\
\hline 19 & 7.56 & Male & Conductive & 4 & 2 \\
\hline 20 & 6.08 & Female & Sensorineural & 4 & 2 \\
\hline 21 & 4.00 & Male & Conductive & 3 & 2 \\
\hline 22 & 7.09 & Male & Conductive & 4 & 2 \\
\hline 23 & 5.27 & Female & Normal & 4 & 2 \\
\hline 24 & 6.50 & Male & Normal & 4 & 2 \\
\hline 25 & 4.01 & Male & Normal & 2 & 2 \\
\hline 26 & 7.42 & Male & Normal & 4 & 2 \\
\hline 27 & 4.47 & Female & Normal & 3 & 2 \\
\hline 28 & 4.97 & Female & Conductive & 4 & 2 \\
\hline 29 & 5.29 & Male & Conductive & 3 & 2 \\
\hline 30 & 6.92 & Male & Conductive & 3 & 2 \\
\hline 31 & 5.95 & Female & Normal & 4 & 2 \\
\hline 32 & 5.95 & Male & Conductive & 2 & 2 \\
\hline 33 & 5.14 & Male & Conductive & 3 & 2 \\
\hline 34 & 5.94 & Female & Normal & 3 & 2 \\
\hline 35 & 4.35 & Male & Sensorineural & 2 & 2 \\
\hline 36 & 5.24 & Male & Sensorineural & 3 & 2 \\
\hline 37 & 7.61 & Male & Sensorineural & 3 & 2 \\
\hline 38 & 5.47 & Female & Sensorineural & 3 & 2 \\
\hline 39 & 4.15 & Female & Sensorineural & 3 & 2 \\
\hline 40 & 7.41 & Female & Sensorineural & 4 & 2 \\
\hline
\end{tabular}

Note: An average balance score of 4 indicates "normal" balance and a nystagmus/oculomotor score of 2 indicates "normal" vestibular function. 
(standard deviation $[\mathrm{SD}]=1.28$ ), with a range of 4 to 8 years. There were 10 children in the $\mathrm{NH}$ group, ages ranging from 4.15 to 7.70 years $($ mean $=6.05$, $\mathrm{SD}=$ 1.23). Nine children were included in the SNHL group, ages ranging from 4.15 to 7.70 (mean $=5.85$, $\mathrm{SD}=$ 1.42). There were 11 children in the OME due to CHL group, ages ranging from 4.0 to 8.42 years (mean = $6.05, \mathrm{SD}=1.31$ ). Table 4 shows the age and gender distribution for each group.

\section{Nystagmus/Oculomotor Test}

For the nystagmus/oculomotor test, a score of 1 was categorized as abnormal, indicated by presence of nystagmus, saccadic eye movements, or disconjugate eye movements. A score of 2 was categorized as normal, indicated by no nystagmus, smoothness of pursuit, and small saccade in direction of movement.

Scores from the nystagmus/oculomotor portion of the functional test were compared among the three hearing status groups. None of the participants in any group displayed spontaneous nystagmus, gaze nystagmus, or post headshake nystagmus. There was, however, a significant difference in the presence of smooth pursuit. The chi-squared test for independence (with Yates Continuity Correlation) was conducted but was found to violate the assumption of chi-square concerning the "minimum expected cell frequency." Therefore, the $\mathrm{NH}$ and SNHL groups were combined to use the Fisher's exact test. The results revealed a significant relationship between hearing status and smooth pursuit $(p=0.012)$. The effect size, described by phi was 0.515 , indicating a large effect size. This indicates that the participants with CHL due to OME displayed abnormal smoothness pursuit significantly more than participants with NH or SNHL. The presence of smoothness of pursuit separated by hearing status is summarized in Table 5.

\section{Balance Test}

There was no statistically significant difference found on any portion of the balance test. Possible scores for the balance test ranged from zero to four points, with four being the maximum number of points. The scale provided by the BOT-2 was used for the scoring. Because of modifications on this portion, the average score of the $\mathrm{NH}$ group was used as the "normal" score in place of the norms provided by the BOT-2.

Balance scores for males and females were significantly different from one another. For all hearing loss conditions, boys had significantly poorer average balance scores $(M=3.13, \mathrm{SD}=0.72)$ than girls $[M=$ $3.71, \mathrm{SD}=0.47 ; t_{(28)}=-2.62, p=0.014$, two-tailed], as illustrated in Figure 1. The differences in means (mean difference $=-0.59,95 \%$ CI: -1.05 to -0.128 ) was small (eta $=0.2$ ). On further analysis, there was a significant difference in gender scores for two tasks: standing on one leg on a line-eyes open and standing on foam. The scores for standing on one leg on a lineeyes open for males $(M=2.56, \mathrm{SD}=1.15)$ were significantly poorer than females $\left[M=3.64, \mathrm{SD}=0.63 ; t_{(23.86)}=\right.$ $-3.23, p=0.004$, two-tailed]. The magnitude of the difference in the means (mean difference $=-1.08,95 \%$ CI: -1.77 to -0.390 ) was small (eta $=0.27$ ). The scores for standing on foam was significantly poorer for males $(M=2.38, \mathrm{SD}=1.31)$ than females $[M=3.21, \mathrm{SD}=$ $0.80 ; t_{(25.25)}=-2.14, p=0.042$, two tailed]. The magnitude of the difference in the means (mean difference $=$ $-0.84,95 \%$ CI: 1.65 to -0.03 ) was small (eta $=0.14$ ). These results indicate that females scored higher on average than males on the balance test, specifically standing on one leg on a line-eyes open and standing on foam.

Balance scores were significantly different as a function of age. The groups were categorized as younger (4 to $<6.5$ years) and older ( $\geq 6.5$ to 8 years $)$ groups. There was a statistically significant difference in overall average balance scores for children $\geq 6.5$ years $(M=3.8$, $\mathrm{SD}=0.42)$ and $<6.5$ years $(M=3.2, \mathrm{SD}=0.7), t_{(28)}=2.5$, $p=0.02$ (two-tailed). The mean difference in scores was 0.6 with a $95 \%$ confidence interval, ranging from 0.11 to 1.10. The eta-squared statistic (0.18) indicated a small effect size. Further analysis revealed there was a significant difference in scores for two tasks. The scores for standing on one leg on a line-eyes closed for the older group $(M=2.5, \mathrm{SD}=1.08)$ were statistically greater than for the younger group $(M=1.65, \mathrm{SD}=0.81)$; $t_{(28)}=2.42, p=0.02$ (two-tailed). The mean difference in scores was 0.85 with a $95 \%$ confidence interval of 0.13 to 1.57 . The eta-squared statistic $(0.17)$ indicated a small effect. In addition, the older cohort $(M=3.4$, $\mathrm{SD}=1.08)$ scored significantly higher than the younger group $(M=2.45, \mathrm{SD}=1.10), t_{(28)}=2.25$, and $p=0.03$

Table 4. The Demographic Information of the Three Groups Included in the Study

\begin{tabular}{llrcr}
\hline Group & Hearing Status & $N$ & Mean Age, in Years (Range) & Female/Male \\
\hline 1 & $\mathrm{NH}$ & 10 & $6.05(4.15-7.70)$ & $7 / 3$ \\
2 & $\mathrm{SNHL}$ & 9 & $5.85(4.15-7.70)$ & $5 / 4$ \\
3 & $\mathrm{CHL}$ (due to OME) & 11 & $6.04(4-8.42)$ & $2 / 9$ \\
Total & & 30 & $5.99(4-8.42)$ & $14 / 16$ \\
\hline
\end{tabular}


Table 5. Presence of Normal and Abnormal Smoothness of Pursuit, Separated by Hearing Status

\begin{tabular}{lccc}
\hline Hearing Status & Total Number & Smooth Pursuit_Normal & Smooth Pursuit-Abnormal \\
\hline $\mathrm{NH}$ & 10 & $10(100 \%)$ & $0(0 \%)$ \\
$\mathrm{SNHL}$ & 9 & $9(100 \%)$ & $0(0 \%)$ \\
$\mathrm{CHL}$ & 11 & $7(64 \%)$ & $4(36 \%)$ \\
\hline
\end{tabular}

Note: Participants with $\mathrm{CHL}$ due to OME were significantly more likely to present with abnormal smoothness of pursuit than those with NH or SNHL. $\chi^{2}=7.972, p=0.005$.

for standing on foam. The magnitude of the difference in the means (mean difference $=0.95,95 \%$ CI: 0.08 to 1.82 ) was small (eta $=0.15$ ). Table 6 summarizes balance scores as a function of age.

\section{Questionnaires}

On the parent questionnaire, the parents of children with CHL due to OME were 3.7 times more likely to report their child complaining of middle ear pressure than the parents of children with SNHL or NH (78\% versus $21 \%$, respectively, $p=0.010$ ). The calculated effect size of 0.542 was considered large, as described by Phi.

On the patient questionnaire, hearing difficulties were reported in $20 \%$ of children with $\mathrm{NH}, 55 \%$ of children with CHL, and $78 \%$ of children with SNHL. Of note, chil- dren with $\mathrm{NH}$ were less likely to report hearing loss than their peers with SNHL but not those with CHL: $F_{(2,27)}=$ $4.89, p=0.038$. The calculated effect size of 0.22 was considered small, according to Cohen's classification.

\section{DISCUSSION}

$\mathrm{T}$ 1 he purpose of this study was to determine whether an efficient vestibular screening could help identify vestibular impairments in children with CHL due to OME. The screening is designed to identify need for diagnostic evaluation.

There were several areas in which children with CHL due to OME differed from children with $\mathrm{NH}$ and SNHL in the present study. Children with OME on average displayed a lack of smooth pursuit more often than

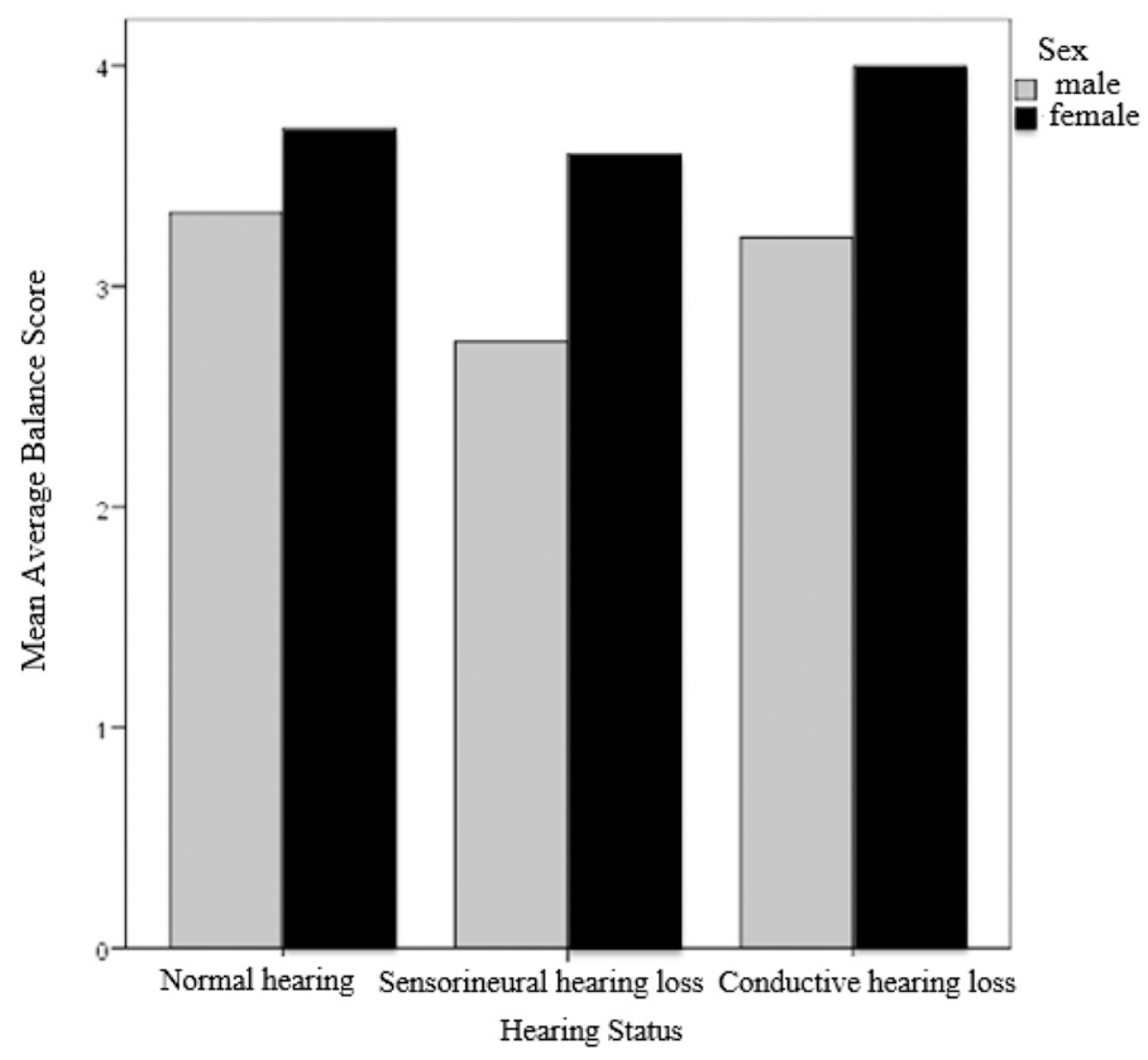

Figure 1. Bar graph demonstrating the difference in mean average balance score between males and females among the three hearing groups. 
Table 6. Balance Scores as a Function of Age, Categorized as Lower Half ( $\leq 6.5$ Years) and Upper Half (>6.5 Years)

\begin{tabular}{|c|c|c|c|c|c|c|}
\hline \multirow[b]{2}{*}{ Task } & \multicolumn{2}{|c|}{ Balance Score (SD) } & \multirow[b]{2}{*}{$t$} & \multirow[b]{2}{*}{$p$ value } & \multirow[b]{2}{*}{ Mean Difference } & \multirow[b]{2}{*}{ Standard Error Difference } \\
\hline & $<6.5$ Years & $>6.5$ Years & & & & \\
\hline Standing with feet apart on a line-eyes open & $3.90(0.45)$ & $4.00(0.00)$ & 0.71 & 0.489 & 0.100 & 0.143 \\
\hline Walk forward on a line & $3.85(0.67)$ & $4.00(0.00)$ & 0.701 & 0.489 & 0.150 & 0.214 \\
\hline Standing on one leg on a line-eyes open & $2.80(1.11)$ & $3.60(0.84)$ & 2.009 & 0.054 & 0.800 & 0.398 \\
\hline Standing with feet apart on a line-eyes closed & $3.95(0.22)$ & $4.00(0.00)$ & 0.701 & 0.489 & 0.050 & 0.071 \\
\hline Walking forward heel-to-toe-eyes open & $3.75(0.79)$ & $3.80(0.63)$ & 0.174 & 0.863 & 0.050 & 0.287 \\
\hline Standing on one leg on a line-eyes closed & $1.65(0.81)$ & $2.50(1.08)$ & 2.419 & $0.022^{*}$ & 0.850 & 0.351 \\
\hline Standing on foam & $2.45(1.10)$ & $3.40(1.07)$ & 2.248 & $0.033^{*}$ & 0.950 & 0.423 \\
\hline Average balance score & $3.20(0.70)$ & $3.80(0.42)$ & 2.494 & $0.019^{*}$ & 0.600 & 0.241 \\
\hline
\end{tabular}

Note: Tasks of standing on one leg on a line-eyes closed, standing on foam, and the average balance score were significantly different between the age cohorts. ${ }^{*} p<0.05$.

those with $\mathrm{NH}$ and SNHL. Parents of children with OME reported a greater incidence of their child reporting middle ear pressure compared with parents of those with NH or SNHL. The patient questionnaire revealed that children with $\mathrm{NH}$ differed significantly from those with SNHL in reporting hearing loss but not significantly from those with CHL from OME. One possible explanation is the mild and transient nature of a CHL.

Although the items on the balance test did not display clinical significance, these tests offered visible observations. Balance is functional, and the items on the BOT-2 subtest displayed balance in a very tangible way. The documentation of objective scores and observations should be included to provide further insight.

With regard to case history, parental report of symptoms and milestones is not always accurate and should be supplemented with objective measures. Many parents admitted to providing approximate timeframes for motor milestones. Parents reported uncertainty about symptoms, including tinnitus, hearing loss, and sensory processing issues. In addition, ambiguity or denial of hearing loss was reported, even in cases of audiometrically confirmed hearing loss. The practitioner must delve deeper into initial responses to get an accurate case history. Previous studies have also reported inaccurate parental reports regarding hearing loss accompanying episodes of OME (Rosenfeld et al, 1998). Objective measures, including hearing evaluation, examination of the middle ear status, and clinical insights, must be used in conjunction with the parental report.

The differences in performance with gender and age suggest the importance of age and gender-specific norms. In this study, females performed, on average, better than males on the functional tests. In addition, older participants scored higher on the functional tests than the younger participants. In particular, the tasks "stand on one leg-eyes closed" and "stand on foam" illustrated the greatest improvement with age. The task, "stand on one leg-eyes open" approached clinical significance and displayed improvement with age. The tests that compromise proprioception draw out the greatest challenges.

\section{CONCLUSION}

B ecause of the high incidence of OME in children and the associated vestibular impairments, a screening measure can identify those in need of diagnostic testing. Based on the present study, children with CHL due to OME appear to experience more oculomotor abnormalities, consistent with vestibular disturbances than their peers with NH. Further research is needed to assess this screening protocol before it is recommended for clinical use.

\section{REFERENCES}

Braswell J, Rine RM. (2006) Evidence that vestibular hypofunction affects reading acuity in children. Int $J$ Pediatr Otorhinolaryngol 70(11):1957-1965.

Bennett KE, Haggard MP, Silva PA, Stewart IA. (2001) Behaviour and developmental effects of otitis media with effusion into the teens. Arch Dis Child 85:91-95.

da Costa Monsanto R, Erdil M, Pauna HF, Kwon G, Schachern PA, Tsuprun V, Paparella MM, Cureoglu S. (2016) Pathologic changes of the peripheral vestibular system secondary to chronic otitis media. Otolaryngol Head Neck Surg 155(3):494-500.

Day BL, Fitzpatrick RC. (2005) The vestibular system. Curr Biol 15(15):R583-R586.

Deitz JC, Kartin D, Kopp K. (2007) Review of the BruininksOseretsky test of motor proficiency, second edition (BOT-2) Phys Occup Ther Pediatr 27(4):87-102.

Deitz JC, Richardson P, Crowe TK, Westcott SL. (1996) Performance of children with learning disabilities and motor delays on the pediatric clinical test of sensory interaction for balance (PCTSIB) Phys Occup Ther Pediatr 16(3):1-21.

Goebel JA. (2001) The ten minute examination of the dizzy patient. Semin Neurol 21(4):391-398.

Gravel JS, Wallace IF. (2000) Effects of otitis media with effusion on hearing in the first 3 years of life. J Speech Lang Hear Res 43(3): 631-644. 
Koyuncu M, Saka M, Tanyeri Y, Seșen T, Unal R, Tekat A, Yilmaz F. (1999) Effects of otitis media with effusion on the vestibular system in children. Otolaryngol Head Neck Surg 120(1):117-121.

McCaslin DL, Jacobson GP, Lambert W, English LN, Kemph AJ. (2015) The development of the vanderbilt pediatric dizziness handicap inventory for patient caregivers (DHI-PC) Int $J$ Pediatr Otorhinolaryngol 79(10):1662-1666.

Picciotti PM, Fiortia A, Di Nardo W, Calo L, Ccarano E, Paludett G. (2007) Vestibular evoked myogenic potentials in children. Int $J$ Pediatr Otorhinolaryngol 71(1):29-33.

Rine RM, Wiener-Vacher S. (2013) Evaluation and treatment of vestibular dysfunction in children. NeuroRehabilitation 32(3):507-518.

Rosenfeld RM, Goldsmith AJ, Madell JR. (1998) How accurate is parent rating of hearing for children with otitis media? Arch Otolaryngol Head Neck Surg 124(9):989-992.

Schaaf RC. (1985) The frequency of vestibular disorders in developmentally delayed preschoolers with otitis media. Am J Occup Ther 39(4):247-252.

Sheykholeslami K, Megerian CA, Arnolda JE, Kaga K. (2005) Vestibular-evoked myogenic potentials in infancy and early childhood. Laryngoscope 115(8):1440-1444.
Soni A. (2006) Ear infections (otitis media) in children (0-17): use and expenditures. Statistical Brief No. 228. www.meps.ahrq.gov/ mepsweb/data_files/publications/st228/stat228.pdf. Accessed June 2017.

Tsuzuku T, Kaga K. (1992) Delayed motor function and results of vestibular function tests in children with inner ear anomalies. Int J Pediatr Otorhinolaryngol 23(3):261-268.

Valente M. (2007) Maturational effects of the vestibular system: a study of rotary chair, computerized dynamic posturography, and vestibular evoked myogenic potentials with children. $J A m$ Acad Audiol 18(6):461-481.

Wiener-Vacher SR, Obeid R, Abou-Elew M. (2012) Vestibular impairment after bacterial meningitis delays infant posturomotor development. J Pediatr 161(2):246-251.e1.

Wolter NE, Cushing SL, Vilchez-Madrigal LD, James AL, Campos J, Papsin BC, Gordon KA. (2016) Unilateral hearing loss is associated with impaired balance in children: a pilot study. Otol Neurotol 37(10):1589-1595.

Zhou G, Dargie J, Dornan B, Whittemore K. (2014) Clinical uses of cervical vestibular-evoked myogenic potential testing in pediatric patients. Medicine 93(4):e37. 